\title{
Exploring the gap in the public's understanding of the links between alcohol and cancer
}

\section{KEYWORDS: Alcoholic beverages, neoplasms, health policy}

Cancer is the leading cause of death in the UK and carries an enormous cost burden, both financial and personal. Around one in four cancers however are lifestyle-related, and therefore ultimately preventable. ${ }^{1}$ These include smoking (15.1\%), being overweight $(6.3 \%)$, ultraviolet radiation (3.8\%), occupation (3.7\%), infections (3.5\%) and alcohol (3.3\%). ${ }^{1}$ Whereas the links between smoking and cancer have been well established and promoted for decades, there has been a failure to communicate similarly important messages about alcohol and obesity. In 2017, a study using the Cancer Awareness Measure validated questionnaire asked 'What things do you think affect a person's chance of developing cancer?', 81.9\% named smoking, 53.5\% alcohol and 36.2\% diet.

This is a significant public health issue. Alcohol accounts for 11,894 cancer cases per year in the UK. ${ }^{1}$ Alcohol use is responsible for $38 \%$ of cancers of the pharynx, $34 \%$ of cancers of the oral cavity, $22 \%$ of cancers of the larynx, $13 \%$ of cancers of oesophagus, $8 \%$ of breast cancers, $7 \%$ of liver cancers and $6 \%$ of gastric cancers. ' While the risk increases with rising levels of consumption, even low levels of drinking carry some degree of risk, particularly for breast cancer. ${ }^{3}$ Whereas the death rates from smoking-related cancers have fallen over the last 20 years, the mortality rates for the majority of alcohol-related cancers have remained static, with an increase in alcohol-related liver cancer deaths. ${ }^{4}$ Furthermore, alcohol-related cancers are strongly and disproportionately linked to health inequalities. ${ }^{5}$

One of the major reasons for the disparity in the public's understanding of alcohol-related cancer risks compared to smoking is likely to be a direct result of the failure of the government to enforce regulation on alcohol advertising, availability and price. Through self-regulation, the alcohol industry (AI) are able to advertise on any media platform, employing effective marketing techniques including the use of celebrities to endorse their products. There is evidence that adolescents are heavily exposed to alcohol advertising via both television and digital media. ${ }^{6,7}$ Drinks companies also sponsor high-profile music and sporting events - Carling sponsors the English football Premier League, Budweiser supports the football World Cup and Guinness were the title sponsor of Six Nations rugby championship in 2019. Alcohol availability has increased year-on-year as a result of relaxed licencing laws, with non-traditional venues such as cinemas and hairdressers now able to sell alcohol. In addition, alcohol affordability has increased by $64 \%$ in the England since $1980,{ }^{8}$ further exacerbated by the removal of the duty escalator in 2014 . Alcohol promotions are commonplace in both the retail environment as well as pubs, bars and clubs. We therefore still live in an 'alcogenic' environment with a lack of regulation that would be unthinkable for smoking. In our opinion, the ubiquitous nature of these adverts and promotions, in addition to the increased availability of alcohol, subtly endorses these products and conflicts with campaigns which highlight alcohol-related harm.

Product labelling is also an issue. Only 14 out of 100 alcoholcontaining products currently display up-to-date labelling reflecting the 2016 low-risk drinking guidelines and none carry any health warnings other than the risks of drinking during pregnancy. ${ }^{9}$ Mass media campaigns aimed at reducing alcohol-related harm, have been both small in number and scale. In 2018, Public Health England (PHE) launched a campaign to encourage middle-aged drinkers to introduce alcohol-free days. The campaign was criticised, however, for its partnership with the industry-funded charity Drinkaware, leading to the resignation of Sir Ian Gilmore who argued that this represented a fundamental conflict of interest. ${ }^{10}$ While the campaign did state that alcohol causes cancer, as far as we are aware, it was the first campaign to do so up to this point.

There is evidence to suggest that in addition to the AI itself, AI funded organisations, usually corporate social responsibility (CSR) bodies (for example the Portman group and Drinkaware), play a critical role in determining the public's understanding of the risks between alcohol and cancer. This occurs on a number of levels, for example the involvement of CSR bodies in alcohol education, policy, prevention programmes and industry funded scientific research. ${ }^{11}$ These organisations have been repeatedly shown to align themselves with the economic interests of the AI rather than acting as independent bodies. ${ }^{11,12}$ Petticrew et al describe three strategies employed by the AI that share strong similarities to those used by tobacco companies when disseminating information about cancer: denying, omitting or disputing the evidence that alcohol consumption increases cancer risk; mentioning cancer, but misrepresenting or obfuscating the nature or size of that risk; and focusing discussion away from the independent effects of alcohol on common cancers. ${ }^{13}$ Concerningly, 24 out of 26 websites from AI-related bodies contained significant omissions or misinformation. The risks between breast cancer and colon cancer were most likely to be misrepresented. ${ }^{13}$ A comparative analysis of twitter feeds from industry funded and non-industry funded charities revealed similar findings, with the AI instead focusing on the behavioural aspects of drinking. ${ }^{12}$ Both the AI and its related bodies have dismissed or disputed the evidence behind the UK 2016 low-risk drinking guidelines, including those that state 'the risk of developing cancers ... increase with any amount you drink on a regular basis.' The Portman Group's reply to the public consultation clearly misrepresents the cancer risk associated with drinking, for example stating that alcohol increases 'the risk of a small number of cancer types', distracting attention away from the number of individuals affected by these cancers. ${ }^{14}$ There is also evidence to suggest 
that by focusing their campaigns on heavy drinkers, the AI have promoted the idea that moderate levels of alcohol consumption carry no additional health risks. ${ }^{15}$ This has erroneously misled alcohol policy towards ensuring that moderate drinkers are unaffected by regulatory measures, and reinforces the acceptance of alcohol corporations within the global governance arena by creating an 'illusion of righteousness'.15,16

There is therefore a strong argument that, globally, we need to move beyond 'tobacco exceptionalism' in which different regulatory approaches are applied towards the tobacco and alcohol industries despite both causing harm. ${ }^{17,18}$ The tobacco industry's past efforts are well documented - a highly successful campaign of evidence denial and false science lasting decades. ${ }^{19}$ Ultimately the industry was exposed and, in 2005, the World Health Organization (WHO) Framework Convention of Tobacco Control came into force. In the UK, public and media pressure from health advocates led the government to implement sanctions as follows: a ban on advertising (2003), sports sponsorship (2005), smoking in public places (2007) and retail displays (2013), and plain packaging removing branding (2016), accompanied by heavy taxation making tobacco $30 \%$ less affordable over the last decade. Mass media cessation campaigns and funding for smoking cessation services were also major contributors in getting this message across. These policies were meaningful and effective, and collectively helped reduce the smoking prevalence for UK adults to $15.1 \%(2017,18+)$, from $26.8 \%$ in $2000(16+) .{ }^{20}$

Non-industry related factors may also challenge the public's understanding of alcohol as a carcinogen and warrant consideration by future public health campaigns. Firstly the 'mechanistic' link between alcohol and cancer compared to smoking may be less obvious. Tobacco enters the upper airways and lungs and therefore it makes sense that it causes throat and lung cancer. Furthermore, carcinomas of the lung and larynx account for over $70 \%$ of smoking-related cancers. ${ }^{1}$ Alcohol causes at least seven types of cancer and, other than liver cancer, the links are by no means obvious. Indeed only $18 \%$ of individuals are aware of a link between alcohol and breast cancer. ${ }^{21}$

The public generally accept that any smoking is bad for health and the advice is simple: 'quit'. For alcohol, the messaging is more complex. The chief medical officer's 2016 low-risk drinking guidelines state for men and women 'It is safest not to drink more than 14 units a week.' The guidelines are set at a level at which the absolute risk of mortality from alcohol is one in 100 people. They also state 'drinking alcohol increases the risk of getting cancers ... these risks start from any level of regular drinking.' There is now robust evidence that low levels of alcohol intake do not provide any protective health benefits (the risk of cancer outweighs any cardiovascular benefits), however, this message may not as yet have reached the general population. ${ }^{22}$ Critically, we are dealing with a spectrum of consumption and risk, where it is possible to consume small amounts of alcohol within the constraints of a healthy lifestyle. This makes the communication of harm far more challenging. Furthermore, public health campaigns have recently highlighted the risks of obesity, a low fibre diet and inactivity with cancer. While this is vitally important, messages that alcohol can also increase the risk of cancer may contribute towards 'information-overload' and individuals may find this too restrictive on their lifestyle.

How do we move forward? The WHO's top three 'best buys' for alcohol policy are all aimed at reducing population level alcohol consumption: increasing excise taxation, a ban on advertising and reduced availability. ${ }^{23}$ These policies target both moderate and harmful drinkers and should therefore be effective at reducing cancer prevalence across this spectrum. Introducing a minimum unit price is not only one of the most effective and cost-effective alcohol policies, but also specifically targets individuals at the greatest risk of alcohol-related harm, including cancer. ${ }^{24}$ This policy has been aggressively opposed by the AI, perhaps because nearly $70 \%$ of their revenue is generated from individuals drinking above the UK's low-risk drinking guidelines. ${ }^{25}$ Minimum unit pricing was introduced in Scotland in 2018 and is undergoing evaluation by the National Welsh Assembly and Northern Ireland, while the Westminster parliament continues to wait.

In our opinion, a complete ban on alcohol advertising is a necessary and measured response to the alcohol-related cancer risk alone. This will be an uphill task in the face of colossal alcohol marketing budgets; however a complete ban, such as the loi Évin introduced in France, would prevent any misleading messages about alcohol-related harm. ${ }^{26}$ In terms of product labelling, we would advocate expansion of the current health warning to include the risks of cancer, liver disease, stroke and mental health problems among others, in addition to clearly stating the 2016 UK low-risk drinking guidelines and nutritional information. While we accept these are likely to be strongly opposed by industry, all of these interventions have been demonstrated to be effective at benefiting consumers. ${ }^{27}$

A significant increase in investment in mass media health campaigns is also required and each campaign needs to be regularly evaluated to ensure it remains effective at altering behaviour. A recent Danish study reported that public health campaigns are both effective at increasing awareness of alcohol as a cancer risk factor and enhance public support for alcohol policies. ${ }^{28}$ Vitally, all public health campaigns must be fully independent of the drinks industry. Unfortunately, as long as the government continues to be influenced by the substantial power of the AI through media such as the Institute of Economic Affairs, ${ }^{29}$ we are likely to be highly dependent on the role of civil society organisations. This year, PHE published new rules of engagement limiting work with commercial operators, which critically adopts the WHO's definition of 'alcohol industry': manufacturers of alcoholic beverages, wholesale distributors, major retailers and importers that deal solely and exclusively in alcohol beverages, or whose primary income comes from trade in alcoholic beverages. ${ }^{30}$ Drinkaware is nearly entirely funded by the AI and therefore we sincerely hope that all government agencies will avoid future collaborations with it, and other CSR bodies. We suggest that public health campaigns focus on communicating that alcohol causes common cancer types, that any level of drinking can increase your cancer risk and that the risk increases the more you drink. Interestingly, the 'truth' campaign which focuses on exposing the duplicitous practices of the tobacco industry to counter their appeal, has proved extremely effective at reducing smoking rates and changing attitudes for young people in America. ${ }^{31}$ We suspect that this approach is likely to be more challenging for alcohol, as the health risks of drinking are less well understood by the public, and the AI and its related bodies are still accepted to the extent that they continue to partner with government organisations and influence policy. There is therefore a significant hill to climb before we reach this point.

In an attempt to find a novel way of communicating the alcoholrelated cancer risks to the public, we recently aimed to harness 
embedded public knowledge about the risks of smoking and cancer and use this as a 'yard-stick', asking the question: 'As far as cancer risk is concerned, how many cigarettes are there in a bottle of wine? ${ }^{32}$ We estimated that for women the risk of drinking 10 units per week is approximately equivalent to 10 cigarettes per week, and five for men. The gender difference results from the fact that the risk of breast cancer is significantly high even at low levels of alcohol consumption. ${ }^{32}$ We hope that these findings will help highlight moderate levels of drinking as an important public health issue for women. This is a message which has so far struggled to reach the public at large, despite being supported by strong scientific evidence for over 15 years. ${ }^{21,33}$

Finally, alcohol cessation programmes require long-term funding commitments to promote their use through increased availability as well as adverts promoting acceptability, in an attempt to replicate the success of the smoking cessation services.

The AI have repeatedly accused such policies of targeting the majority for diseases that affect a minority. Cancer affects one in two individuals in the UK and kills one in four of us. ${ }^{34}$ Around $60 \%$ of UK adults drink regularly, and $14 \%$ of women and $28 \%$ of men drink above 14 units a week. ${ }^{35}$ Furthermore, $64 \%$ of the population are overweight or obese. ${ }^{35}$ This means that many individuals are suffering from both lifestyle issues which are known to act synergistically in terms of disease development and progression. Arguments regarding the revenue generated by the AI needs to be balanced against the fact that alcohol misuse is estimated to cost $£ 21$ billion a year for England and Wales. ${ }^{36}$ We therefore hope that our government will make the necessary moves in the fight against cancer by prioritising the public's health above their relationship with this powerful industry.

Theresa J Hydes

Gastroenterology registrar, University Hospital Southampton NHS Foundation Trust, Southampton, UK

Roger Williams

Professor of hepatology, Foundation for Liver Research, London, UK and King's College London, London, UK

Nick Sheron

Professor of hepatology, Foundation for Liver Research, London, UK

\section{References}

1 Brown KF, Rumgay H, Dunlop C et al. The fraction of cancer attributable to modifiable risk factors in England, Wales, Scotland, Northern Ireland, and the United Kingdom in 2015. Br J Cancer 2018:118:1130-41.

2 Cancer Intelligence Team. Cancer awareness measure 2017. Cancer Research UK, 2017.

3 Cao Y, Willett WC, Rimm EB, Stampfer MJ, Giovannucci EL. Light to moderate intake of alcohol, drinking patterns, and risk of cancer: results from two prospective US cohort studies. BMJ 2015;351:h4238.

4 Institute for Health Metrics and Evaluation. Global burden of disease data visualizations. University of Washington, 2017.

5 Anderson P, Baumberg B. Alcohol in Europe: A Public health perspective. London: Institute of Alcohol Studies, 2006.

6 Patil S, Winpenny EM, Elliott MN, Rohr C, Nolte E. Youth exposure to alcohol advertising on television in the UK, the Netherlands and Germany. Eur ] Public Health 2014;24:561-5.

7 Lobstein T, Landon J, Thornton N, Jernigan D. The commercial use of digital media to market alcohol products: a narrative review. Addiction 2017;112:21-7.
8 National Statistics. Statistics on alcohol, England 2019. NHS Digital, 2019.

9 Panorama. Britain's drink problem. BBC, 2019.

10 Gilmore I, Bauld L, Britton J. Public Health England's capture by the alcohol industry. BMJ 2018;362:k3928.

11 Babor T, Robaina K. Public health, academic medicine, and the alcohol industry's corporate social responsibility activities. Am J Public Health 2013;103:206-14.

12 Maani Hessari N, van Schalkwyk M, Thomas S, Petticrew M. Alcohol industry CSR organisations: what can their twitter activity tell us about their independence and their priorities? A comparative analysis. Int J Environ Res Public Health 2019;16:892.

13 Petticrew M, Maani Hessari N, Knai C, Weiderpass E. How alcohol industry organisations mislead the public about alcohol and cancer. Drug Alcohol Rev 2018;37:293-303.

14 Portman Group. Portman Group response to: How to keep health risks from drinking alcohol to a low level: public consultation on proposed new guidelines. Response document 11. Department of Health, 2016:95-101. https://assets.publishing.service.gov.uk/ government/uploads/system/uploads/attachment_data/ file/545757/Response_document_11.pdf.

15 Casswell S. Vested interests in addiction research and policy. Why do we not see the corporate interests of the alcohol industry as clearly as we see those of the tobacco industry? Addiction 2013;108:680-5.

16 Yoon S, Lam T-H. The illusion of righteousness: corporate social responsibility practices of the alcohol industry. BMC Public Health 2013;13:630.

17 McCambridge J, Morris S. Comparing alcohol with tobacco indicates that it is time to move beyond tobacco exceptionalism. Eur ] Public Health 2019;29:200-1.

18 Hawkins B, Holden C, Eckhardt J, Lee K. Reassessing policy paradigms: A comparison of the global tobacco and alcohol industries. Glob Public Health 2018;13:1-19.

19 Proctor RN. The history of the discovery of the cigarette-lung cancer link: evidentiary traditions, corporate denial, global toll. Tob Control 2012;21:87-91.

20 National Statistics. Statistics on smoking, England 2018. NHS Digital, 2018.

21 Buykx P, Li J, Gavens L et al. An investigation of public knowledge of the link between alcohol and cancer. University of Sheffield and Cancer Research UK, 2015.

22 Griswold MG, Fullman N, Hawley C et al. Alcohol use and burden for 195 countries and territories, 1990-2016: a systematic analysis for the Global Burden of Disease Study 2016. Lancet. 2018;392:1015-35.

23 World Health Organization. 'Best buys' and other recommended interventions for the prevention and control of noncommunicabe diseases: Updated (2017) appendix 3 of the global action plan for the prevention and control of noncommunicable diseases 2013 2020. Geneva: WHO, 2017.

24 Sheron N, Chilcott F, Matthews L, Challoner B, Thomas M. Impact of minimum price per unit of alcohol on patients with liver disease in the UK. Clin Med 2014;14:396-403.

25 Bhattacharya A, Angus C, Pryce R et al. How dependent is the alcohol industry on heavy drinking in England? Addiction 2018;113:2225-32.

26 Regaud A, Craplet M. The 'Loi Evin': a French exception. The Globe 2004;2.

27 Martin-Moreno JM, Harris ME, Breda J et al. Enhanced labelling on alcoholic drinks: reviewing the evidence to guide alcohol policy. Eur J Public Health 2013;23:1082-7.

28 Christensen ASP, Meyer MKH, Dalum P, Krarup AF. Can a mass media campaign raise awareness of alcohol as a risk factor for cancer and public support for alcohol related policies? Prev Med (Baltim) 2019;126:105722.

29 Gornall J. Big tobacco, the new politics, and the threat to public health. BMJ 2019;365:I2164.

30 Public Health England. Principles for engaging with industry stakeholders. PHE, 2019. www.gov.uk/government/publications/ 
principles-for-engaging-with-industry-stakeholders/principles-forengaging-with-industry-stakeholders.

31 Farrelly MC, Davis KC, Haviland ML, Messeri P, Healton CG. Evidence of a dose-response relationship between "truth" antismoking ads and youth smoking prevalence. Am J Public Health 2005;95:425-31.

32 Hydes T], Burton R, Inskip H, Bellis MA, Sheron N. A comparison of gender-linked population cancer risks between alcohol and tobacco: how many cigarettes are there in a bottle of wine? BMC Public Health 2019;19:316.

33 Hamajima N, Hirose K, Tajima K et al. Alcohol, tobacco and breast cancer-collaborative reanalysis of individual data from 53 epidemiological studies, including 58,515 women with breast cancer and 95,067 women without the disease. $\mathrm{Br}$ ] Cancer 2002;87:1234-45.
34 Cancer Research UK. Cancer Statistics for the UK. Cancer Research UK. www.cancerresearchuk.org/health-professional/cancerstatistics-for-the-uk

35 National statistics. Health survey for England 2017. NHS Digital, 2018.

36 Williams R, Alexander G, Armstrong I et al. Disease burden and costs from excess alcohol consumption, obesity, and viral hepatitis: fourth report of the Lancet Standing Commission on Liver Disease in the UK. Lancet 2018;391:1097-107.

Address for correspondence: Dr Theresa Hydes, Department of Gastroenterology and Hepatology, University Hospital Southampton NHS Foundation Trust, Tremona Road, Southampton SO16 6YD, UK.

Email: therasa@doctors.org.uk

\section{Royal College} of Physicians

\section{Advancing medical professionalism}

Articulating a modern professional identity helps doctors to understand and undertake the unique role they play in healthcare. Developed in consultation with healthcare professionals, patients and other stakeholders, this report from the Royal College of Physicians aims to help doctors improve their professionalism in practical ways.

It focuses on seven key aspects of professional practice:

$>$ doctor as healer

$>$ patient partner

$>$ learner and teacher

$>$ team worker

$>$ advocate

$>$ manager and leader

$>$ innovator

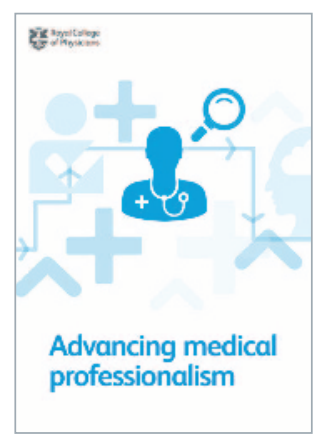

Download the report at: www.rcplondon.ac.uk/advancing-medical-professionalism 